\title{
Light Attenuation Changes with Photo-Acclimation in a Culture of Synechocystis sp. PCC6803
}

Levi Straka $^{\mathrm{a}}$ and Bruce E. Rittmann ${ }^{1}$

${ }^{a}$ Swette Center for Environmental Biotechnology, The Biodesign Institute at Arizona State University, P.O. Box 875701, Tempe, Arizona 85287-5701, U. S. A.

Declaration of author's contributions:

Levi Straka designed the experiments, collected the data, and did the primary analysis, interpretation, and reporting of the data. Bruce Rittmann provided further insight for data analysis, interpretation, and reporting. Both wrote the manuscript cooperatively.

Corresponding Author: Levi Straka (1straka@asu.edu) 


\section{Abstract and Graphical Abstract}

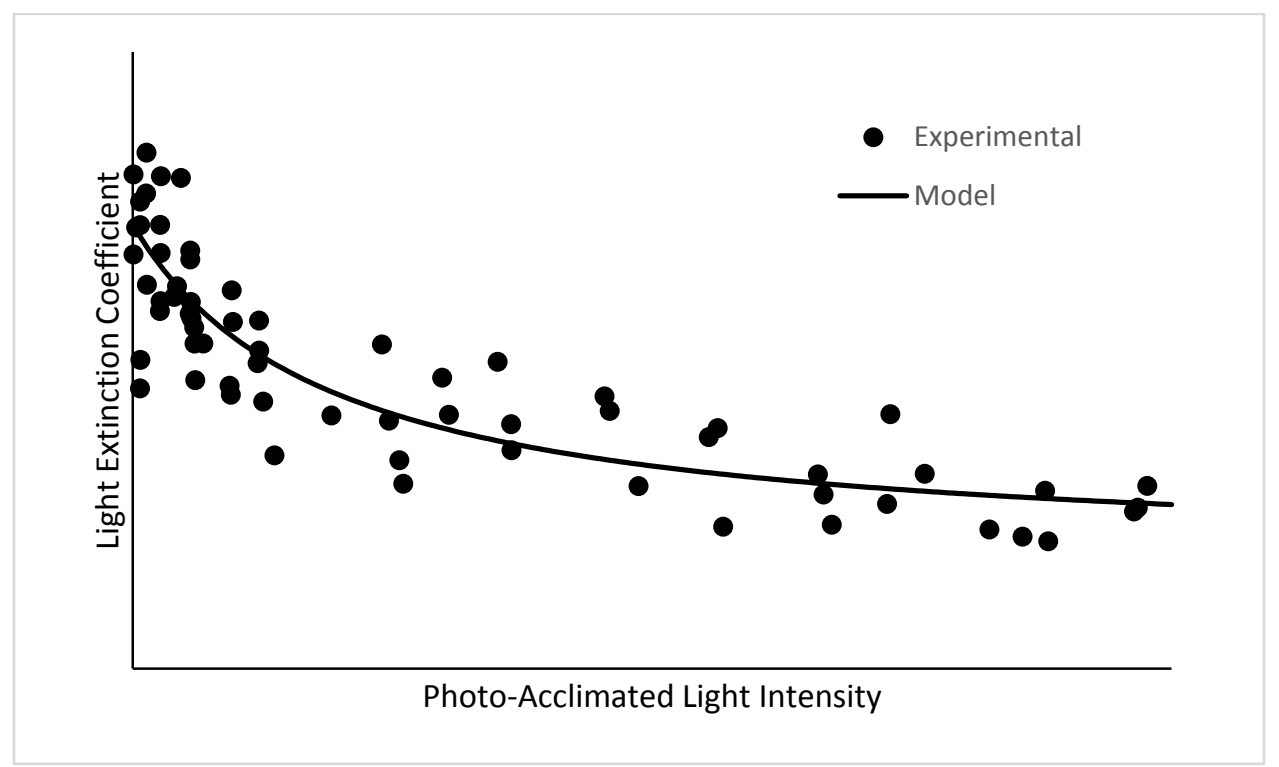

An inherent complication in the relationship between light intensity (LI) and microalgae growth rate is that light attenuates through a culture due to its absorption by biomass. While a biomass's specific extinction coefficient ( $\varepsilon$ describing how rapidly light attenuates) often is assumed to be a constant for a species for mathematical modeling, it is well documented that pigmentation and light absorption depend on growth conditions, particularly light intensity itself. In this study using Synechocystis sp. PCC 6803, we investigated the effect of LI on $\varepsilon$. Using cultures fully acclimated to the LI at which they were grown, we found that biomass grown at higher LI absorbed less light than biomass grown at lower LI; thus, $\varepsilon$ was larger for lower LI. We quantify the relationship between $\varepsilon$ and the acclimated LI and suggest that $\varepsilon$ would be an appropriate metric for describing photo-acclimation.

\section{Keywords:}

Synechocystis sp. PCC6803; mathematical modeling; photo-acclimation; extinction coefficient; light-limited kinetics 


\section{Introduction}

Mathematical modeling of microalgal growth is a valuable tool for optimizing biomass productivity in engineered systems and understanding primary production in natural systems [1]. A growth-limiting factor that has received much attention is light, because its intensity varies naturally, and light is the energy source driving photosynthesis [2]. Unlike a growth-limiting nutrient, light attenuates through a microalgal culture, which means that the light intensity (LI) declines away from its source. Understanding light attenuation is important, because it controls the LI available to cells within the culture and because light attenuation is partly caused by lightenergy absorption of the biomass [3,4].

Light attenuation is most often represented mathematically by the Beer-Lambert equation, which utilizes an exponential function based on the light path (d, in $\mathrm{m})$, biomass concentration $\left(X\right.$, in $\left.\mathrm{mg} \cdot \mathrm{L}^{-1}\right)$, and an extinction coefficient $\left(\varepsilon\right.$, in $\left.\mathrm{m}^{2} \cdot \mathrm{g}^{-1}\right)$ to represent the light absorption of the microalgae culture $[2,5]$ :
$\mathrm{LI}=\mathrm{LI}_{0} \exp (-\varepsilon \mathrm{Xd})$
Equation 1

where LI is the local light intensity $\left(\mu \mathrm{mol} \cdot \mathrm{m}^{-2} \cdot \mathrm{s}^{-1}\right)$, and $\mathrm{LI}_{0}$ is the incident light intensity $\left(\mu \mathrm{mol} \cdot \mathrm{m}^{-2} \cdot \mathrm{s}^{-1}\right)$. In practice, $\varepsilon$ is empirically determined and accounts for the aggregate effect of light scattering and light absorption [4]. While in-depth analyses of the radiative properties of microalgae suggest that scattering is an important phenomenon in microalgae LI extinction, they also report that the vast majority (about 99.9\%) of scattering occurs in the forward direction [69]. Forward-scattered light can be absorbed by other biomass; thus, only back-scattered light at the light's entering surface and light scattered out of the edges of the reactor are truly lost to scattering. 
Light absorption depends on the pigmentation of the biomass, while light scattering depends on cell morphology and surface properties [10]. It is well established that microalgae change their pigmentation and cell morphology in response to different light conditions as a component of photo-acclimation [10-12]. Microalgae also change their pigmentation in response to adverse growth conditions, such as nutrient limitation, non-optimal salinity, or extreme LI [13]. However, the common practice in modeling light attenuation is to assume a constant $\varepsilon$ for a given species of microalgae $[2,14-17]$. Although the phenomena of changing $\varepsilon$ with growth conditions has not received much attention, past research with Chlorella vulgaris demonstrated that $\varepsilon$ depended on culture biomass density $[5,18]$.

A metric that has been used to identify the photo-acclimation state in microalgae growth models is the ratio of chlorophyll-to-carbon $[11,19,20]$. For most species, however, the biomass contains significant amounts of non-chlorophyll pigments, and the primary pigments in cyanobacteria are phycobilisomes, not chlorophylls $[11,21,22]$. For these reasons, chlorophyll content is not a good metric for absorbance or photo-acclimation. Alternatively, we suggest that $\varepsilon$ is a better metric than the chlorophyll-to-carbon ratio for identifying the photo-acclimation state, as it accounts for the aggregate effect of all pigment and morphological changes.

In this study, we use the cyanobacterium Synechocystis sp. PCC6803 (simply Synechocystis from here) to test the hypothesis that the LI to which microalgae are acclimated systematically affects its ability to absorb light. In particular, photo-acclimation affects $\varepsilon$ such that biomass grown at low LI has a higher $\varepsilon$ than biomass grown at high LI. This finding also suggests that $\varepsilon$ can be an appropriate parameter to represent photo-acclimation. 


\section{Materials and Methods}

Synechocystis was grown in a Photobioreactor FMT150 (Photon Systems Instruments, Drásov, Czech Republic; simply FMT from here) with nominal incident light settings from 0 to $6626 \mu \mathrm{mol} \cdot \mathrm{m}^{-2} \cdot \mathrm{s}^{-1}$ of Photosynthetically Active Radiation (PAR), a liquid volume of $370 \mathrm{~mL}$, and a fixed temperature of $30^{\circ} \mathrm{C}$. The FMT is described in detail by Nedbal et. al. (2008) [23]. We replaced the factory-supplied diffusor with an Aquarium Fine Bubble Air Stone (Top Fin ${ }^{\circledR}$, Phoenix, AZ), and air was supplied by an EcoPlus ${ }^{\circledR}$ aquarium air pump at approximately 0.1 $\mathrm{L} \cdot \mathrm{min}^{-1}$ (Sunlight Supply, Inc., Vancouver, WA) and filtered through a 0.2- $\mu \mathrm{m}$ membrane bacterial air vent (Pall Corporation, Ann Arbor, MI). The FMT took automatic readings of optical density at $735 \mathrm{~nm}\left(\mathrm{OD}_{735}\right)$ and $680 \mathrm{~nm}\left(\mathrm{OD}_{680}\right)$. The operating conditions utilized the Turbidostat Module, which added fresh growth medium using a peristaltic pump when the $\mathrm{OD}_{735}$ reached an upper set value, and it stopped delivering medium when it reached a lower set value. We set the $\mathrm{OD}_{735}$ range at 0.20 to 0.21 . The $\mathrm{pH}$ was controlled using an $\mathrm{MC} 122 \mathrm{pH}$ Controller (Milwaukee Instruments, Rocky Mount, NC), which opened a solenoid valve (Milwaukee Instruments, Rocky Mount, $\mathrm{NC}$ ) to bubble pure $\mathrm{CO}_{2}$ into the reactor when the $\mathrm{pH}$ exceeded 8.5 maintaining a $\mathrm{pH}$ between 7.5 and 8.6. Growth medium was autoclaved standard BG-11 as described by Rippka et al. [24]. The FMT cultivation vessel was autoclaved and inoculated from a flask seed culture.

The FMT had nominal light settings ranging from 0 to $6626 \mu \mathrm{mol} \cdot \mathrm{m}^{-2} \cdot \mathrm{s}^{-1} \mathrm{PAR}$. To determine the actual incident light intensities, we used a LI-190 PAR sensor (LI-COR Biosciences, Lincoln, NE) and measured the light entering the cultivation vessel directly behind a piece of glass placed where the inside wall of the FMT cultivation vessel would be. We measured 9 positions equally spaced over the irradiated area (Figure S1 in Supplemental 
Information) and at 23 different nominal light settings ranging from 0 to $3200 \mu \mathrm{mol} \cdot \mathrm{m}^{-2} \cdot \mathrm{s}^{-1} \mathrm{PAR}$. The LI was not uniform, with higher light intensity in the center and less intensity towards the top and bottom of the vessel (Figure S2). We computed an area-weighted average of the nine points to provide an average incident light reading at each of the light settings tested. The result was a calibration between the nominal FMT light setting $\left(\mathrm{LI}_{\mathrm{FMT}}\right)$ to the actual average incident light intensity $\left(\mathrm{LI}_{0}\right): \mathrm{LI}_{0}=2.06 *\left(\mathrm{LI}_{\mathrm{FMT}}-81.2\right)^{0.826}\left(\right.$ standard error $\left.=8.86 \mu \mathrm{mol} \cdot \mathrm{m}^{-2} \cdot \mathrm{s}^{-1}\right)$.

Because the biomass concentration was relatively dilute and the $\mathrm{OD}_{735}$ range within the FMT narrow, we used the average $\mathrm{LI}\left(\mathrm{LI}_{\mathrm{ave}}\right)$ as an approximation of the photo-acclimated $\mathrm{LI}$ $\left(\mathrm{LI}_{\mathrm{acc}}\right)$ of the culture. $\mathrm{LI}_{\mathrm{ave}}$ was computed as an area integration of the Beer-Lambert equation: $\begin{array}{ll}\mathrm{LI}_{\mathrm{ave}}=\frac{\mathrm{LI}_{0}(1-\exp (-\varepsilon \mathrm{Xw}))}{\varepsilon X \mathrm{w}} & \text { Equation } 2\end{array}$ where $\mathrm{w}$ is the width of the bioreactor $(0.024 \mathrm{~m}$ for the FMT).

We independently determined $\varepsilon$ by taking a 20 -ml culture sample and placing it in a $60-$ mm x 15-mm petri dish (VWR®, Radnor, PA) with a liquid depth of $9 \mathrm{~mm}$. The sample was illuminated from underneath with a 54-W fluorescent lamp (Hydrofarm, Inc., Petaluma, CA), and the light intensity was measured above the sample using the LI-190 PAR sensor. The sample was then diluted and measured again. Once 5 different dilutions (100\% sample, $80 \%$, $60 \%, 40 \%$, and $20 \%$ ) and a water control were measured, the data were used to determine $\varepsilon$ of the sample by fitting the $\mathrm{X}$ and LI data to the Beer-Lambert Equation (Equation 1), where $\mathrm{d}$ was $0.009 \mathrm{~m}$, and $\mathrm{LI}_{0}$ was approximately $320 \mu \mathrm{mol} \cdot \mathrm{m}^{-2} \cdot \mathrm{s}^{-1}$ (the reading for water) (Figure S3). Dry

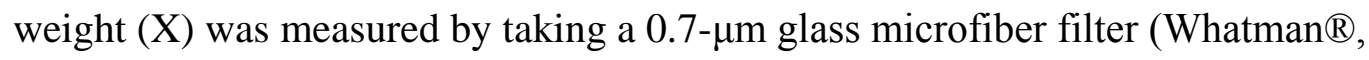
Buckinghamshire, UK) and drying it overnight at $60^{\circ} \mathrm{C}$, weighing it, filtering $10 \mathrm{ml}$ of culture through it, drying it at $60^{\circ} \mathrm{C}$ overnight again, and subtracting the mass before from the mass after. During this study the biomass dry weight concentration was between $94-177 \mathrm{mg} \cdot \mathrm{L}^{-1}$. 
All $\varepsilon$ values are reported for steady-state operating conditions, which we determined after $\mathrm{OD}_{680} / \mathrm{OD}_{735}$ and growth (as determined by time between dilutions) were stable. Due to turbidostat operation, the dilution rate was tied to growth rate, which was between 1.5 and $2.5 \mathrm{~d}^{-1}$ for $\mathrm{LI}_{\mathrm{ave}}>125 \mu \mathrm{mol} \cdot \mathrm{m}^{-2} \cdot \mathrm{s}^{-1}$ and as low as $0.25 \mathrm{~d}^{-1}$ at $\mathrm{LI}_{\mathrm{ave}}=13 \mu \mathrm{mol} \cdot \mathrm{m}^{-2} \cdot \mathrm{s}^{-1}$. Light acclimation, however, was independent of dilution rate. When the previous LI was lower than the LI being considered, steady-state typically was achieved 2 to 3 days after changing the light, and it took about 1 day when the previous LI was higher. Each measured $\varepsilon$, along with the corresponding X and $\mathrm{LI}_{0}$, was used to compute $\mathrm{LI}_{\text {ave }}$ for that point using Equation 2.

The reactor vessel periodically had visible biofilm and floc formation, particularly at higher $\mathrm{LI}_{\mathrm{ave}}$. When this occurred, we removed the culture from the reactor vessel, scrubbed the vessel with bleach and Alconox® cleaner (Alconox, Inc., White Plains, NY), rinsed it thoroughly, filtered the culture through sterile cheese cloth, and returned the culture to the vessel. We discarded all data collected when the FMT contained visible biofilms or flocculated biomass.

All curve fittings, including the $\varepsilon$ determinations described above, and all best-fit parameters in Equation 3 (below) were obtained by least-squares fitting between the experimental and modeled results, and standard errors were calculated [25]. The plot of residuals was generated by subtracting $\varepsilon$ predicted from Equation 3 from the measured $\varepsilon$. 


\section{Results and Discussion}

Measured $\varepsilon$ values for $\mathrm{LI}_{\mathrm{ave}}$ (assumed to be equal to the photo-acclimated $\mathrm{LI}$; $\mathrm{LI}_{\mathrm{acc}}$ ) with dilute biomass concentrations are displayed in

Figure 1 , which clearly shows that $\varepsilon$ was not constant. Instead, $\varepsilon$ declined from its maximum $\left(\varepsilon_{\max }=\sim 0.18 \mathrm{~m}^{2} \cdot \mathrm{g}^{-1}\right)$ at very low $\mathrm{LI}_{\mathrm{acc}}$ and stabilized at a minimum level of approximately 0.045 $\mathrm{m}^{2} \cdot \mathrm{g}^{-1}\left(\varepsilon_{\mathrm{min}}\right)$ as $\mathrm{LI}_{\mathrm{acc}}$ becomes very large. We mathematically represent the systematic changes in $\varepsilon$ using:

$\varepsilon=\frac{\left(\varepsilon_{\max }-\varepsilon_{\min }\right) k_{\varepsilon}}{k_{\varepsilon}+L I_{a c c}}+\varepsilon_{\min }$ Equation 3

where $\mathrm{k}_{\varepsilon}$ is the half maximum light absorption $\mathrm{LI}_{\mathrm{acc}}\left(\mu \mathrm{mol} \cdot \mathrm{m}^{-2} \cdot \mathrm{s}^{-1}\right), \varepsilon_{\max }$ is the measured $\varepsilon$ value at the smallest $\mathrm{LI}_{\mathrm{acc}}$ able to sustain net positive growth, and $\varepsilon_{\min }$ is extrapolated from the $\varepsilon$ trend as $\mathrm{LI}_{\mathrm{acc}}$ approaches infinity. The best-fit values for the experimental data are summarized in Table 1 and were used to produce the model line in Figure 1. 


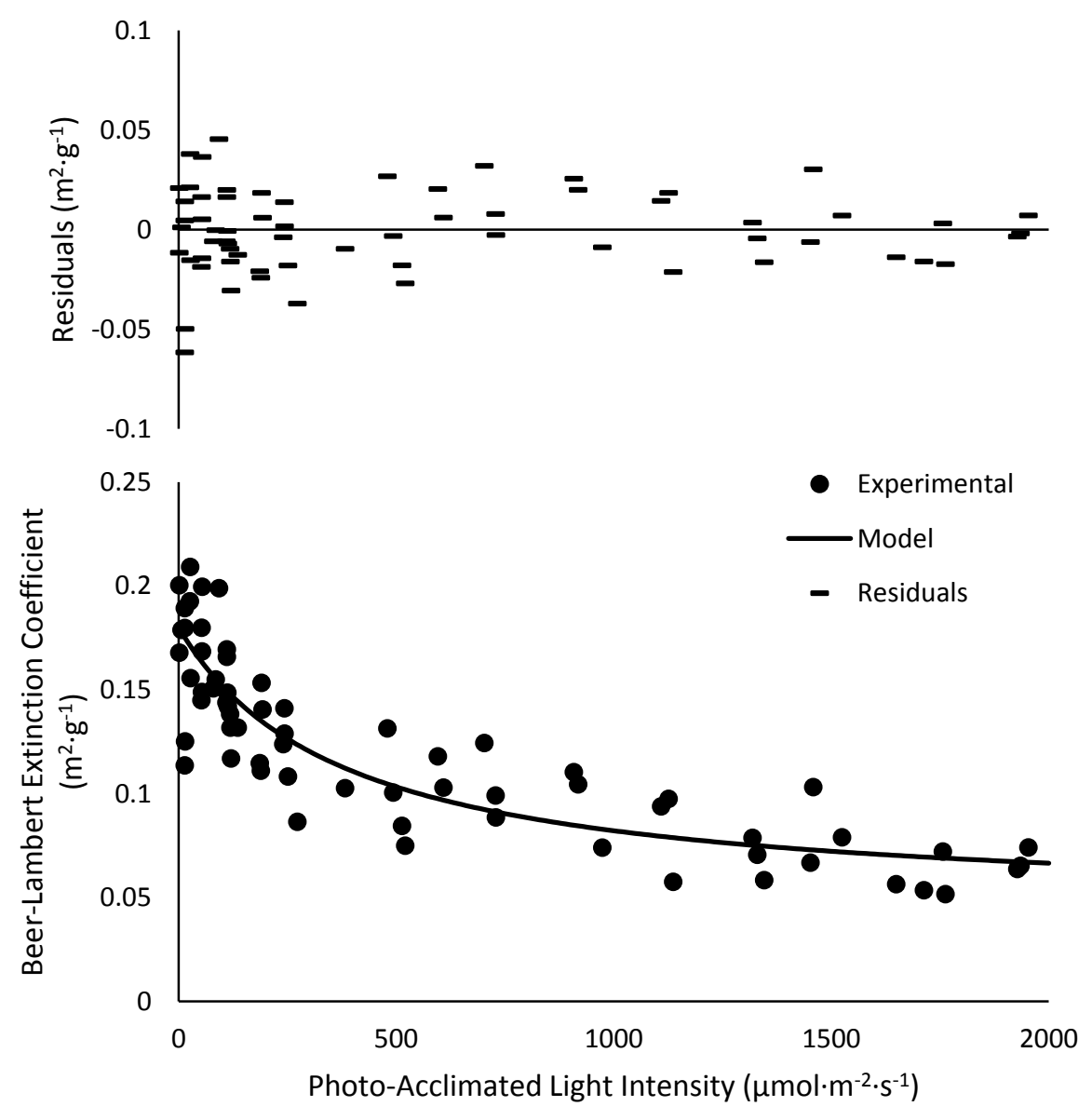

Figure 1: Beer-Lambert extinction coefficient ( $\varepsilon$; lower plot) as a function of photo-acclimated light intensity $\left(\mathrm{LI}_{\mathrm{acc}}\right)$ determined with steady-state dilute cultures of Synechocystis in an FMT photobioreactor at different average internal light intensities (assumed to be $\mathrm{LI}_{\mathrm{acc}}$ ). The data are modeled (lower plot) using Equation 3 with the parameters in Table 1 (standard error for $\varepsilon=$ $0.0070 \mathrm{~m}^{2} \cdot \mathrm{g}^{-1}$ ), and residuals are presented in the upper plot.

Table 1: Best-fit parameters for the light-dependent Beer-Lambert extinction coefficient (equation 3)

\begin{tabular}{|c|l|c|c|}
\hline Parameter & Description & value & units \\
\hline$\varepsilon_{\max }$ & $\begin{array}{l}\varepsilon \text { for biomass photo-acclimated to the lowest possible } \\
\mathrm{LI}_{\text {acc }}\end{array}$ & 0.18 & $\mathrm{~m}^{2} \cdot \mathrm{g}^{-1}$ \\
\hline$\varepsilon_{\min }$ & $\begin{array}{l}\varepsilon \text { for biomass without any light absorption, only } \\
\text { scattering }\end{array}$ & 0.045 & $\mathrm{~m}^{2} \cdot \mathrm{g}^{-1}$ \\
\hline $\mathrm{k}_{\varepsilon}$ & Half-maximum-absorption LI & 380 & $\mu \mathrm{mol} \cdot \mathrm{m}^{-2} \cdot \mathrm{s}^{-1}$ \\
\hline
\end{tabular}

The value of $\varepsilon$ was most sensitive to $\mathrm{LI}_{\mathrm{acc}}$ in the region of lower $\mathrm{LI}_{\mathrm{acc}}$, where $\varepsilon$ increased steadily as $\mathrm{LI}_{\mathrm{acc}}$ declined. The higher $\varepsilon$ at low $\mathrm{LI}_{\text {acc }}$ also was qualitatively apparent by the culture 
appearing greener than cultures grown at higher LI. This trend supports that Synechocystis maximized light absorption when light was scarce by increasing light-absorbing pigments. At the other end of the $\mathrm{LI}_{\mathrm{acc}}$ range, the $\varepsilon$ value changed proportionally less as $\mathrm{LI}_{\text {acc }}$ increased to 2000 $\mu \mathrm{mol} \cdot \mathrm{m}^{-2} \cdot \mathrm{s}^{-1}$. This trend is similar to chlorophyll measurements taken for Chlorella $[11,19]$ and for Synechocystis [26] (although quantitatively quite different for Synechocystis, as chlorophyll is only one component of photo-acclimation). The residuals plot of Figure 1 demonstrates that variability of the measured $\varepsilon$ was random throughout the range of $\mathrm{LI}_{\mathrm{acc}}$ tested, although the magnitude of the variability was slightly larger for lower $\mathrm{LI}_{\mathrm{acc}}$. This trend is expected, because $\varepsilon$ is more sensitive to changes in LI at lower LI.

Another metric that correlates to pigment content is the ratio of $\mathrm{OD}_{680}$, which accounts for absorption of red light, to $\mathrm{OD}_{735}$, which is beyond the spectrum of light absorbed for photosynthesis and therefore a measure of turbidity or light scattering. An increase in $\mathrm{OD}_{680} / \mathrm{OD}_{735}$ signifies an increase in light-absorbing pigment. Comparing $\mathrm{OD}_{680} / \mathrm{OD}_{735}$ to $\varepsilon$ in Figure 2 makes it apparent that they increase linearly with each other at a slope of approximately $0.16 \varepsilon /\left(\mathrm{OD}_{680} / \mathrm{OD}_{735}\right)$ 


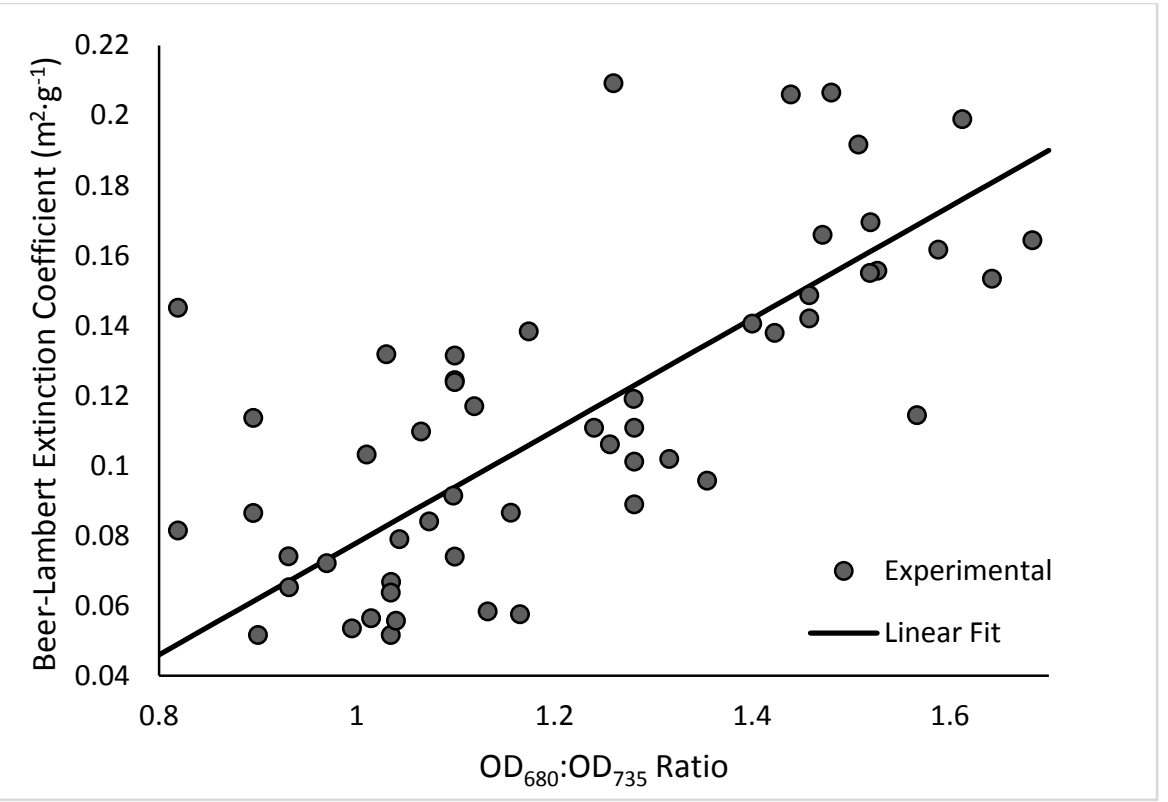

Figure 2: Beer-Lambert extinction coefficient ( $($ ) correlated to the ratio of Synechocystis $\mathrm{OD}_{735} / \mathrm{OD}_{680}$, representing the increase in pigment concentration related to light extinction. The linear regression has a slope of 0.16 and y-intercept of -0.082 (standard error for $\varepsilon=0.0044$ $\left.\mathrm{m}^{2} \cdot \mathrm{g}^{-1}, \mathrm{R}^{2}=0.47\right)$.

At very high $\mathrm{LI}_{\mathrm{acc}}, \varepsilon_{\min }$ theoretically represents $\varepsilon$ for only light scattering. For this condition, the biomass should have minimal light-absorbing pigments, and we can consider the fraction of $\varepsilon$ due to absorbance negligible. Using light microscopy, we observed minor changes in cell size in response to photo-acclimation to the range of LI values (Figure S4); changes in cell size would suggest changes in light scattering at changing $\mathrm{LI}_{\mathrm{acc}}$. However, the strong trend in Figure 2 between $\varepsilon$ and $\mathrm{OD}_{680} / \mathrm{OD}_{735}$ suggests that the changes in $\varepsilon$ are dominated by absorbance, and, therefore, $\varepsilon_{\min }$ (reflecting scattering) can be assumed to be a constant (assuming constant reactor geometry and $\mathrm{X}$ ). The amount of light absorbed is then given by the difference between total extinction and scattering: $\left(\varepsilon-\varepsilon_{\min }\right) * \mathrm{LI}_{\text {ave }}$.

The shape of the $\varepsilon$ curve with respect to $\mathrm{LI}_{\mathrm{acc}}$ is roughly inverse to a Michaelis-Menton or Monod-type function, which is sometimes used to represent the effects of LI (or a soluble substrate) on growth kinetics $[2,27]$. The increase in $\varepsilon$ supports that Synechocystis was mounting 
a physiological response to counteract the normal decline in growth rate with lowered LI. These countering trends in $\mathrm{LI}_{\mathrm{acc}}$ and $\varepsilon$ suggest that Synechocystis growth kinetics may be best modeled

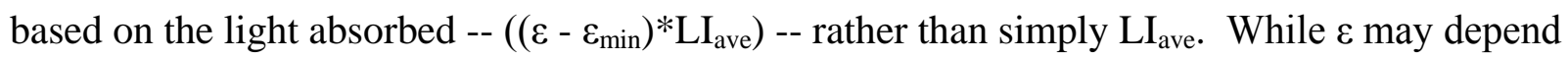
on other factors (e.g., nutrient concentrations and light spectrum; our white LEDs differ from sunlight), LI acclimation had a significant and systematic impact on $\varepsilon$. Thus, $\varepsilon$ is an appropriate and useful metric for the biomass's photo-acclimation state.

\section{Conclusion}

The Beer-Lambert extinction coefficient ( $\varepsilon$ ) of Synechocystis sp. PCC6805 changed significantly and systematically in response to photo-acclimation to a wide range of light intensities. Specifically, $\varepsilon$ was highest for biomass grown at lower $\operatorname{LI}_{\text {acc }}\left(\sim 0.18 \mathrm{~m}^{2} \cdot \mathrm{g}^{-1}\right)$ and stabilized at a much lower level $\left(\sim 0.045 \mathrm{~m}^{2} \cdot \mathrm{g}^{-1}\right)$ when $\mathrm{LI}_{\mathrm{acc}}$ was at very high values (up to 2000 $\left.\mu \mathrm{mol} \cdot \mathrm{m}^{-2} \cdot \mathrm{s}^{-1}\right)$. The increase in $\varepsilon$ at low $\mathrm{LI}_{\mathrm{acc}}$ appears to be a physiological response to counteract the normal decline in growth rate with lowered $\mathrm{LI}_{\mathrm{acc}}$, and this is supported by the increase in $\mathrm{OD}_{680} / \mathrm{OD}_{735}$. Measuring $\varepsilon$ may be an appropriate metric to represent the photo-acclimated state of the biomass. 


\section{References}

[1] T. Iwakuma, M. Yasuno, A comparison of several mathematical equations describing photosynthesis-light curve for natural phytoplankton populations., Arch. Fur Hydrobiol. Stuttgart. (1983). http://scholar.google.com/scholar?hl=en\&btnG=Search\&q=intitle:A+comparison+of+seve ral+mathematical+equations+describing+photosynthesislight+curve+for+natural+phytoplankton+populations\#0 (accessed November 6, 2014).

[2] Q. Béchet, A. Shilton, B. Guieysse, Modeling the effects of light and temperature on algae growth: state of the art and critical assessment for productivity prediction during outdoor cultivation., Biotechnol. Adv. 31 (2013) 1648-63. doi:10.1016/j.biotechadv.2013.08.014.

[3] L. Pottier, J. Pruvost, J. Deremetz, J.F. Cornet, J. Legrand, C.G. Dussap, A fully predictive model for one-dimensional light attenuation by Chlamydomonas reinhardtii in a torus photobioreactor, Biotechnol. Bioeng. 91 (2005) 569-582. doi:10.1002/bit.20475.

[4] J.-F. Cornet, C.G. Dussap, J.-B. Gros, C. Binois, C. Lasseur, A simplified monodimensional approach for modeling coupling between radiant light transfer and growth kinetics in photobioreactors, Chem. Eng. Sci. 50 (1995) 1489-1500. doi:10.1016/0009-2509(95)00022-W.

[5] Y.-S. Yun, J.. Park, Attenuation of monochromatic and polychromatic lights in Chlorella vulgaris suspensions, Appl. Microbiol. Biotechnol. 55 (2001) 765-770. doi: $10.1007 / \mathrm{s} 002530100639$.

[6] H. Berberoglu, L. Pilon, Experimental measurements of the radiation characteristics of Anabaena variabilis ATCC 29413-U and Rhodobacter sphaeroides ATCC 49419, Int. J. Hydrogen Energy. 32 (2007) 4772-4785. doi:10.1016/j.ijhydene.2007.08.018.

[7] H. Berberoglu, L. Pilon, A. Melis, Radiation characteristics of Chlamydomonas reinhardtii CC125 and its truncated chlorophyll antenna transformants tla1, tlaX and tla1-CW+, Int. J. Hydrogen Energy. 33 (2008) 6467-6483. doi:10.1016/j.ijhydene.2008.07.071.

[8] R.-L. Heng, E. Lee, L. Pilon, Radiation characteristics and optical properties of filamentous cyanobacterium Anabaena cylindrica., J. Opt. Soc. Am. A. Opt. Image Sci. Vis. 31 (2014) 836-45. http://www.ncbi.nlm.nih.gov/pubmed/24695147.

[9] K.G. Privoznik, K.J. Daniel, F.P. Incropera, Absorption, extinction and phase function measurements for algal suspensions of chlorella pyrenoidosa, J. Quant. Spectrosc. Radiat. Transf. 20 (1978) 345-352. doi:10.1016/0022-4073(78)90103-6.

[10] R. Kandilian, E. Lee, L. Pilon, Radiation and optical properties of Nannochloropsis oculata grown under different irradiances and spectra, Bioresour. Technol. 137 (2013) 6373. doi:10.1016/j.biortech.2013.03.058.

[11] H.L. MacIntyre, T.M. Kana, T. Anning, R.J. Geider, Photoacclimation of Photosynthesis Irradiance Response Curves and Photosynthetic Pigments in Microalgae and Cyanobacteria, J. Phycol. 38 (2002) 17-38. 
http://onlinelibrary.wiley.com/doi/10.1046/j.1529-8817.2002.00094.x/full (accessed November 7, 2014).

[12] D. Talmy, J. Blackford, N. Hardman-Mountford, A.J. Dumbrell, R.J. Geider, An optimality model of photoadaptation in contrasting aquatic light regimes, Limnol. Oceanogr. 58 (2013) 1802-1818. doi:10.4319/lo.2013.58.5.1802.

[13] K.J.M. Mulders, P.P. Lamers, D.E. Martens, R.H. Wijffels, Phototrophic pigment production with microalgae: biological constraints and opportunities, J. Phycol. 50 (2014) 229-242. doi:10.1111/jpy.12173.

[14] R. Bosma, E. van Zessen, J.H. Reith, J. Tramper, R.H. Wijffels, Prediction of Volumetric Productivity of an Outdoor Photobioreactor, Biotechnol. Bioeng. 97 (2007) 1108-1120. doi:10.1002/bit.

[15] J.U. Grobbelaar, C.J. Soeder, E. Stengel, Modeling algal productivity in large outdoor cultures and waste treatment systems, Biomass. 21 (1990) 297-314. doi:10.1016/01444565(90)90079-Y.

[16] A. Muller-Feuga, R. Le Guédes, J. Pruvost, Benefits and limitations of modeling for optimization of Porphyridium cruentum cultures in an annular photobioreactor, J. Biotechnol. 103 (2003) 153-163. doi:10.1016/S0168-1656(03)00100-7.

[17] H.W. Kim, R. Vannela, C. Zhou, C. Harto, B.E. Rittmann, Photoautotrophic nutrient utilization and limitation during semi-continuous growth of Synechocystis sp. PCC6803., Biotechnol. Bioeng. 106 (2010) 553-63. doi:10.1002/bit.22724.

[18] Q. Béchet, P. Chambonnière, A. Shilton, G. Guizard, B. Guieysse, Algal productivity modeling: A step toward accurate assessments of full-scale algal cultivation., Biotechnol. Bioeng. 112 (2015) 987-96. doi:10.1002/bit.25517.

[19] J. Myers, Culture Conditions And The Development Of The Photosynthetic Mechanism. III. Influence of Light Intensity on Cellular Characteristics of Chlorella, J. Gneral Physiol. 29 (1946) 419-427.

[20] C. Zonneveld, Modeling effects of Photoadaption on the Photosynthesis-Irradiance Curve, J. Theor. Biol. 186 (1997) 381-8. http://www.ncbi.nlm.nih.gov/pubmed/25089930.

[21] S. Akimoto, M. Yokono, E. Yokono, S. Aikawa, A. Kondo, Short-term light adaptation of a cyanobacterium, Synechocystis sp. PCC 6803, probed by time-resolved fluorescence spectroscopy, Plant Physiol. Biochem. 81 (2014) 149-154.

doi:10.1016/j.plaphy.2014.01.007.

[22] A. Morel, A. Bricaud, Theoretical Results Concerning Light-Absorption in a Discrete Medium, and Application To Specific Absorption of Phytoplankton, Deep. Res. Part aOceanographic Res. Pap. 28 (1981) 1375-1393. doi:10.1016/0198-0149(81)90039-X.

[23] L. Nedbal, M. Trtílek, J. Červený, O. Komárek, H.B. Pakrasi, A photobioreactor system for precision cultivation of photoautotrophic microorganisms and for high-content analysis of suspension dynamics, Biotechnol. Bioeng. 100 (2008) 902-910. 
doi:10.1002/bit.21833.

[24] R. Rippka, J. Deruelles, J.B. Waterbury, M. Herdman, R.Y. Stanier, Generic Assignments, Strain Histories and Properties of Pure Cultures of Cyanobacteria, J. Gen. Microbiol. 111 (1979) 1-61. doi:10.1099/00221287-111-1-1.

[25] T. Hastie, R. Tibshirani, J. Friedman, The Elements of Statistical Learning: Data Mining, Inference, and Prediction, 2nd ed., Springer, 2009. doi:10.1007/b94608.

[26] D. Stramski, A. Morel, Optical properties of photosynthetic picoplankton in different physiological states as affected by growth irradiance, Deep Sea Res. Part A, Oceanogr. Res. Pap. 37 (1990) 245-266. doi:10.1016/0198-0149(90)90126-G.

[27] J. Monod, The Growth of Bacterial Cultures, Annu. Rev. Microbiol. 3 (1949) 371-394. 